\title{
A fitted finite element method for the numerical approximation of void electro-stress migration
}

\author{
Robert Nürnberga ${ }^{\mathrm{a}}$, Andrea Sacconi ${ }^{\mathrm{a}, *}$ \\ ${ }^{a}$ Department of Mathematics, Imperial College, London, SW7 2AZ, UK
}

\begin{abstract}
Microelectronic circuits usually contain small voids or cracks, and if those defects are large enough to sever the line, they cause an open circuit. A fully practical finite element method for the temporal analysis of the migration of voids in the presence of surface diffusion, electric loading and elastic stress is presented. We simulate a bulk-interface coupled system, with a moving interface governed by a fourth-order geometric evolution equation and a bulk where the electric potential and the displacement field are computed. The method presented here follows a fitted approach, since the interface grid is part of the boundary of the bulk grid. A detailed analysis, in terms of experimental order of convergence (when the exact solution to the free boundary problem is known) and coupling operations (e.g., smoothing/remeshing of the grids, intersection between elements of the two grids), is carried out. A comparison with a previously introduced unfitted approach (where the two grids are totally independent) is also performed, along with several numerical simulations in order to test the accuracy of the methods.
\end{abstract}

\section{Introduction}

Microelectronic circuits contain thin lines of aluminium alloy, that make electric contact between neighbouring devices possible. These lines are passivated with a layer of oxide at large temperatures, and during the cooling process large stresses are induced. As the dimensions of microchips are reduced further and further, and since interconnects always contain small voids or cracks, it is of great interest to investigate the physical mechanisms that impede such a reduction, due to mechanical failures in the lines induced by the motion of the cracks. The problem analysed in this paper involves the evolution over time of voids in a conducting metal line where three different contributions to the drift of the voids are present: the surface tension, the electric field and the elastic energy. This phenomenon is known as electro-stress migration; for further details see, e.g., [36, 14, 3], and the references therein.

As the height of interconnect lines is much smaller than the dimensions of the base, voids generally fully penetrate the conducting material. Hence it is common to consider a two dimensional model for void electro-stress migration, and this is the approach that we are going to pursue in this paper. In addition, for ease of exposition, we assume that the interconnect line is given by a rectangular solid. The electric field is induced in the line by prescribing the voltage on its vertical boundaries, while the displacement field is induced by prescribing the stresses on its four boundaries.

In this paper, based on our previous work in [31], we introduce a novel front-tracking, fitted finite element method for the approximation of void electro-stress migration. The main difference to the approximation presented in [31] is that here we consider the fitted approach, which means that the interface mesh is always part of the boundary of the bulk grid. Moreover, we also include the effect of stress-migration into the model. As an aside we note that our method inherits the good interface mesh properties from the approximation in [31]. In particular, the vertices on the discrete interface equidistribute asymptotically so that no reparameterisation of the discrete interface is necessary in practice.

The paper is organised as follows. In Section 2 we give a mathematical description of the problem of void electro-stress migration that we are interested in. We also give a brief overview of the different

\footnotetext{
${ }^{*}$ Corresponding author

Email addresses: robert.nurnberg@imperial.ac.uk (Robert Nürnberg), a.sacconi11@imperial.ac.uk (Andrea Sacconi)
} 
numerical methods applicable to this problem. In addition, we highlight the differences between the fitted approach presented in this paper and the unfitted approach previously introduced by the authors in [31]. Section 3 contains a detailed description of our proposed finite element approximation. In Section 4 we discuss possible solution methods of the algebraic system of equations arising at each time level. In addition, we present details on the bulk mesh smoothing strategy. Finally, in Section 5 we perform a convergence experiment for a test case in which the exact solution is known, and we present various other examples of the application of our numerical method.

\section{Problem formulation}

For the formulation of the governing equations we closely follow the presentation in [31], see also [3]. Let $\Omega=\left(-L_{1}, L_{1}\right) \times\left(-L_{2}, L_{2}\right)$, where $L_{1}, L_{2}>0$, be the domain that contains the conductor. We denote the boundary of $\Omega$ with $\partial \Omega$. At any time $t \in[0, T]$, let $\Gamma(t) \subset \Omega$ be the boundary of the void $\Omega_{-}(t)$ inside the conductor $\Omega$. Then $\Gamma(t)=\partial \Omega_{-}(t)$ and $\Omega_{+}(t):=\Omega \backslash \overline{\Omega_{-}(t)}$ denotes the conducting region (see Figure 1). Now the evolution of the interface $\Gamma(t)$, which represents the void boundary, is given by

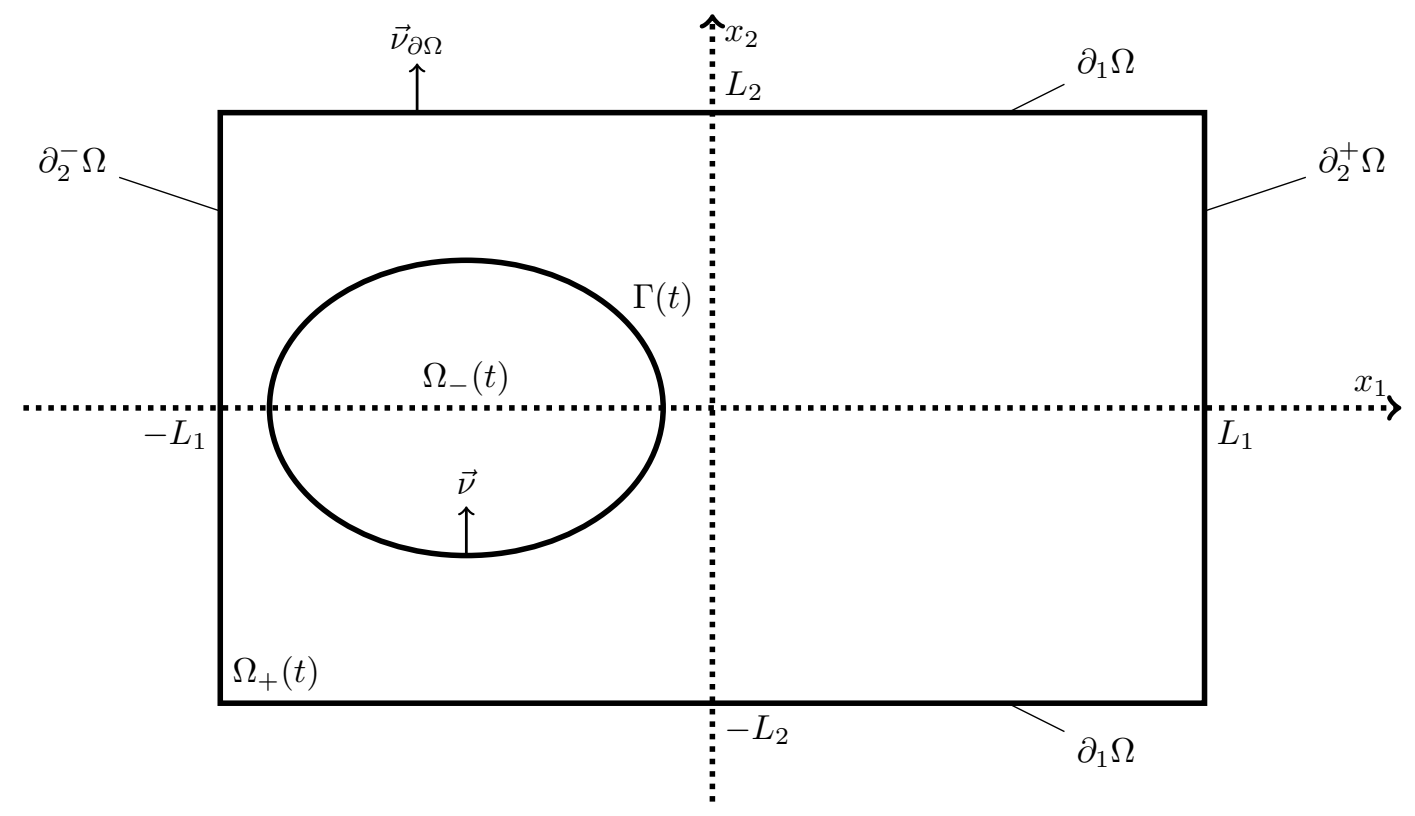

Figure 1: The domain $\Omega$ and the void with its boundary $\Gamma(t)$.

$$
\mathcal{V}=-\alpha_{1} \varkappa_{s s}+\alpha_{2} \phi_{s s}+\alpha_{3}(E(\vec{u}))_{s s}
$$

where $\mathcal{V}$ represents the velocity of $\Gamma(t)$ in the direction $\vec{\nu}$ (the unit normal to $\Gamma(t)$ pointing into $\Omega_{-}(t)$ ), $s$ is the arc-length of the curve, $\varkappa$ is the curvature of $\Gamma(t)$ (positive when $\Omega_{-}(t)$ is convex). In particular, it holds that

$$
\vec{x}_{s s}=\varkappa \vec{\nu}
$$

where $\vec{x}$ is a suitable parameterisation of $\Gamma(t)$, i.e. $\Gamma(t)=\vec{x}(I, t)$, with $I=\mathbb{R} / \mathbb{Z}$ denoting the "periodic" interval $[0,1]$.

The second contribution on the right-hand side of $(1)$ is given by the electric potential $\phi(t)$, which satisfies a Laplace equation in $\Omega_{+}(t)$, i.e.:

$$
\begin{array}{cccc}
\triangle \phi=0 \quad \text { in } \Omega_{+}(t), & \frac{\partial \phi}{\partial \vec{\nu}}=0 & \text { on } \Gamma(t), \\
\frac{\partial \phi}{\partial \vec{\nu}_{\partial \Omega}}=0 \text { on } \partial_{1} \Omega, & \phi=g^{ \pm} & \text {on } \partial_{2}^{ \pm} \Omega,
\end{array}
$$


where $\vec{\nu}_{\partial \Omega}$ is the outer normal to $\partial \Omega$. In (3b), $g^{ \pm}:= \pm L_{1}$ denotes the Dirichlet boundary condition on parts of $\partial \Omega$, where $\partial \Omega=\partial_{1} \Omega \cup \partial_{2} \Omega$, with $\partial_{1} \Omega \cap \partial_{2} \Omega=\emptyset$ and

$$
\partial_{2} \Omega=\partial_{2}^{-} \Omega \cup \partial_{2}^{+} \Omega \quad \text { with } \quad \partial_{2}^{ \pm} \Omega:=\left\{ \pm L_{1}\right\} \times\left[-L_{2}, L_{2}\right] .
$$

The Dirichlet boundary conditions in (3b) model a uniform parallel electric field, $\phi \approx x_{1}$ as $L_{1} \rightarrow \infty$.

The third contribution on the right-hand side of (1) is given by the elastic energy density $E(\vec{u})$, where $\vec{u}$ is the displacement field. Precisely,

$$
E(\vec{u}):=\frac{1}{2} \mathcal{C} \underline{\underline{\mathcal{E}}}(\vec{u}): \underline{\underline{\mathcal{E}}}(\vec{u})
$$

where

$$
\underline{\underline{\mathcal{E}}}(\vec{u}):=\frac{1}{2}\left(\nabla \vec{u}+(\nabla \vec{u})^{T}\right)
$$

is the symmetric strain tensor and $\mathcal{C}$ is the possibly anisotropic elasticity tensor, which we assume to be symmetric and positive definite. For the sake of notation, recall that the inner product $\underline{\underline{\mathcal{A}}}: \underline{\underline{\mathcal{B}}}$ of two matrices $\underline{\underline{\mathcal{A}}}, \underline{\underline{\mathcal{B}}} \in \mathbb{R}^{2 \times 2}$ is defined as $\sum_{i, j=1}^{2} \mathcal{A}_{i j} \mathcal{B}_{i j}$. In addition, the trace of a tensor $\underline{\underline{\mathcal{A}}}$ is denoted by $\operatorname{Tr}(\underline{\underline{\mathcal{A}}}):=\mathcal{A}_{11}+\mathcal{A}_{22}$, and the divergence is defined as $\nabla \cdot \underline{\underline{\mathcal{A}}}=\left(\frac{\partial \mathcal{A}_{11}}{\partial x_{1}}+\frac{\partial \mathcal{A}_{12}}{\partial x_{2}}, \frac{\partial \mathcal{A}_{21}}{\partial x_{1}}+\frac{\bar{\partial} \mathcal{A}_{22}}{\partial x_{2}}\right)^{T}$. We will assume throughout for all $i, j, k, l \in\{1,2\}$ that

$$
\text { (i) } \quad \mathcal{C}_{i j k l}=\mathcal{C}_{j i k l}=\mathcal{C}_{i j l k} \quad \text { and } \quad(i i) \quad \mathcal{C}_{i j k l}=\mathcal{C}_{k l i j}
$$

Here $(i)$ follows, without loss of generality, from the fact that $\mathcal{C}$ maps symmetric tensors to symmetric tensors; and (ii) follows from the symmetry assumption $\mathcal{C} \underline{\underline{\mathcal{A}}}: \underline{\underline{\mathcal{B}}}=\underline{\underline{\mathcal{A}}}: \mathcal{C} \underline{\underline{B}}$. We assume also throughout that $\mathcal{C}$ is positive definite; that is, there exist constants $m_{\mathcal{C}}, M_{\mathcal{C}}$ such that

$$
0<m_{\mathcal{C}}(\underline{\underline{\mathcal{A}}}: \underline{\underline{\mathcal{A}}}) \leq \mathcal{C} \underline{\underline{\mathcal{A}}}: \underline{\underline{\mathcal{A}}} \leq M_{\mathcal{C}}(\underline{\underline{\mathcal{A}}}: \underline{\underline{\mathcal{A}}}) \quad \forall \underline{\underline{\mathcal{A}}} \in \mathbb{R}^{2 \times 2} \backslash\{\underline{\underline{0}}\} .
$$

If one further assumes cubic symmetry, then it also follows that $\mathcal{C}_{1111}=\mathcal{C}_{2222}$ and $\mathcal{C}_{2212}=\mathcal{C}_{1112}=0$; see, e.g., [20]. For an isotropic material we obtain that

$$
\mathcal{C} \underline{\underline{\mathcal{E}}}(\vec{u})=2 \mu \underline{\underline{\mathcal{E}}}(\vec{u})+\lambda \operatorname{Tr}(\underline{\underline{\mathcal{E}}}(\vec{u})) \underline{\underline{\mathcal{I}}},
$$

where $\underline{\underline{\underline{I}}}$ is the identity tensor, and $\mu \in \mathbb{R}_{>0}$ and $\lambda \in \mathbb{R}_{\geq 0}$ are the Lamé moduli. The unknown $\vec{u}$ is the displacement field which is the solution to the following problem:

$$
\begin{array}{lll}
\nabla \cdot(\mathcal{C} \underline{\underline{\mathcal{E}}}(\vec{u})) & =\overrightarrow{0} & \text { in } \Omega_{+}(t), \\
\mathcal{C} \underline{\underline{\mathcal{E}}}(\vec{u}) \vec{\nu} & =\overrightarrow{0} & \text { on } \Gamma(t), \\
\mathcal{C} \underline{\underline{\mathcal{E}}}(\vec{u}) \vec{\nu}_{\partial \Omega}=\vec{g} & \text { on } \partial \Omega .
\end{array}
$$

The function $\vec{g} \in L^{2}(\partial \Omega)$ is the given boundary force satisfying the necessary compatibility conditions, $\int_{\partial \Omega} \vec{g} \mathrm{~d} s=\overrightarrow{0}$ and $\int_{\partial \Omega} \vec{g} \cdot\left(x_{2},-x_{1}\right)^{T} \mathrm{~d} s=0$ for the existence of a solution $\vec{u}$ to (7). For simplicity, we will consider

$$
\vec{g}=\underline{\underline{\mathcal{S}}} \vec{\nu}=\mathcal{C} \underline{\underline{\mathcal{S}}}^{*} \vec{\nu}
$$

where $\underline{\underline{\mathcal{S}}} \in \mathbb{R}^{2 \times 2}$ is a symmetric tensor and $\underline{\underline{\mathcal{S}}}^{*}:=\mathcal{C}^{-1} \underline{\underline{\mathcal{S}}}$. Alternatively, one could prescribe displacement boundary conditions, $\vec{u}=\vec{f}$ on $\partial \Omega$ or on parts thereof.

We should note that the solution $\vec{u}$ to $(7)$ is not unique. This is simply because

$$
\underline{\underline{\mathcal{E}}}(\vec{v})=\overrightarrow{0} \quad \forall \vec{v} \in \underline{\mathrm{RM}},
$$

where $\underline{\mathrm{RM}}$ is the space of rigid motions and characterised by

$$
\underline{\mathrm{RM}}:=\left\{\vec{v} \in \underline{H}^{1}(\Omega): \vec{v}=\vec{p}+q\left(x_{2},-x_{1}\right)^{T}, \vec{p} \in \mathbb{R}^{2}, q \in \mathbb{R}\right\} .
$$

Hence one can impose uniqueness for (7) by seeking $\vec{u}$ such that $\int_{\Omega_{+}(t)} \vec{u} \cdot \vec{v} \mathrm{~d} x=0$ for all $\vec{v} \in \underline{\mathrm{RM}}$. 
Finally, $\alpha_{1} \in \mathbb{R}_{>0}$ and $\alpha_{2}, \alpha_{3} \in \mathbb{R}_{>0}$ are given parameters depending on the conductor, on the strength of the electric field and on the magnitude of the elastic stress. The first term on the right-hand side of (1) is surface diffusion due to interfacial tension, which models atoms moving around the boundary of the void to positions of large curvature, whereas the second and third term are surface diffusion due to the electric field and the elastic energy, respectively. The void electro-stress migration model is then the coupled system of equations (1), (3), and (7). In the case $\alpha_{2}=\alpha_{3}=0$, the evolution (1) is called surface diffusion, which is an example of a geometric evolution equation. A local existence result for the motion by surface diffusion can be found in [18]. Moreover, it was shown that a global solution exists if the initial curve, $\Gamma(0)$, is close to a circle and that it converges to a circle. Numerical approximations of surface diffusion have been proposed in $[2,4,6]$, and we refer to [17] for a review of possible numerical approaches for the approximation of geometric evolution equations in general. For $\alpha_{2}, \alpha_{3} \geq 0$, the motion (1) preserves the area enclosed by the closed curve $\Gamma(t)$ since

$$
\frac{d}{d t}\left|\Omega_{-}(t)\right|=-\int_{\Gamma(t)} \mathcal{V} \mathrm{d} s=0,
$$

where $|D|$ is the measure of a domain $D$. In addition, for $\alpha_{2}=0$ the system has a Lyapunov structure. In fact, it can be shown that

$$
\begin{aligned}
& \frac{d}{d t}\left[\alpha_{1}|\Gamma(t)|+\alpha_{3}\left(\int_{\Omega_{+}(t)} E(\vec{u}) \mathrm{d} x-\int_{\partial \Omega} \vec{g} \cdot \vec{u} \mathrm{~d} s\right)\right] \\
& \quad=-\alpha_{1} \int_{\Gamma(t)} \mathcal{V} \varkappa \mathrm{d} s+\alpha_{3} \int_{\Omega_{+}(t)} \mathcal{C} \underline{\underline{\mathcal{E}}}(\vec{u}): \underline{\underline{\mathcal{E}}}\left(\vec{u}_{t}\right) \mathrm{d} x+\alpha_{3} \int_{\Gamma(t)} \mathcal{V} E(\vec{u}) \mathrm{d} s-\alpha_{3} \int_{\partial \Omega} \vec{g} \cdot \vec{u}_{t} \mathrm{~d} s \\
& \quad=-\int_{\Gamma(t)} \mathcal{V}\left(\alpha_{1} \varkappa-\alpha_{3} E(\vec{u})\right) \mathrm{d} s=-\int_{\Gamma(t)}\left(\alpha_{1} \varkappa_{s}-\alpha_{3}[E(\vec{u})]_{s}\right)^{2} \mathrm{~d} s \leq 0 .
\end{aligned}
$$

As a special case, we recover for $\alpha_{2}=\alpha_{3}=0$ the well-known result that surface diffusion decreases the length of the interface, since

$$
\frac{d}{d t}|\Gamma(t)|=-\int_{\Gamma(t)} \mathcal{V} \varkappa \mathrm{d} s=-\alpha_{1} \int_{\Gamma(t)}\left(\varkappa_{s}\right)^{2} \mathrm{~d} s \leq 0
$$

It turns out that our numerical approximation will satisfy a discrete analogue of (13), while it does not appear possible to derive a finite element approximation that also satisfies the stronger analogue of (12).

For later use we recall the following true solution of a circular void, moving at constant speed through an infinite conductor. That is, for any $\alpha_{1}, \alpha_{2} \in \mathbb{R}_{\geq 0}, \alpha_{3}=0, R \in \mathbb{R}_{>0}$, and $z=\left(z_{1}, z_{2}\right) \in \mathbb{R}^{2}$,

$$
\Gamma(t):=\left\{x \in \mathbb{R}^{2}:\left(x_{1}-z_{1}(t)\right)^{2}+\left(x_{2}-z_{2}\right)^{2}=R^{2}\right\}, \quad z_{1}(t):=z_{1}+\frac{2 \alpha_{2}}{R} t,
$$

where the corresponding electric potential

$$
\phi(x, t)=\left[x_{1}-z_{1}(t)\right]\left(1+\frac{R^{2}}{\left(x_{1}-z_{1}(t)\right)^{2}+\left(x_{2}-z_{2}\right)^{2}}\right)
$$

solves (1) and (3) with

$\Omega_{+}(t)$ in (3a) replaced by $\mathbb{R}^{2} \backslash \overline{\Omega_{-}(t)}$ and (3b) replaced by $\nabla \phi \rightarrow(1,0)^{T}$ as $|x| \rightarrow \infty$.

Observe that (1) reduces to $\mathcal{V}=-\frac{2 \alpha_{2}}{R^{2}}\left[x_{1}-z_{1}(t)\right]$ on $\Gamma(t)$. The explicit solution (14a), (14b) was first noted in [21].

The void electro-stress migration problem (1), (3) and (7) represents a complicated free boundary problem which couples the evolution of an interface to quantities that satisfy partial differential equations in the bulk. For the numerical approximation of such free boundary problems several different approaches are possible. In phase field methods, the sharp interface is replaced by a diffusive interfacial layer, in which a phase field variable rapidly changes between two different constant values that represent the void and the conductor, respectively. We refer to $[27,28,12,9,3,5]$ for different phase field approximations 
of void electro-migration and stress-migration. Also in the level set method, see [32], the interface is only treated implicitly. We refer to $[26,1]$ and $[35, \S 18.5]$ for examples of the application of the level set method to the approximation of void electro-migration. Finally, in front-tracking methods the interface is discretised directly and is moved by the approximated normal velocity of the interface. This requires an explicit coupling to the approximations of the bulk quantities. Examples of such methods for the approximation of void electro-migration and stress-migration can be found in [13, 25, 36, 31]. In this paper, based on our work in [31], we propose a new front-tracking method for void electro-stress migration that will be based on a parametric finite element description of the moving interface.

Recall that in [31] an unfitted finite element approach was used, where the bulk mesh and the parametric interface mesh were totally independent. As a consequence, no smoothing of the bulk mesh needed to be performed. Moreover, an equidistribution property for the vertices of the interface mesh could be shown. However, the implementation of the unfitted approach requires a delicate communication between the two grids, and a simple approximation of (3a) leads to additional approximation errors. See [31] for further details. Below we list the main features of the fitted approach considered in this paper:

- only the exterior of the interface is triangulated, and so there is no necessity of filtering the bulk grid to identify a subset of elements on which the bulk problems are solved;

- the vertices of the interface grid can be easily identified within the bulk mesh by a mapping, which needs to be computed only once at the beginning;

- the interpolation of finite element functions over the bulk to the interface can be easily done with the previous mapping.

However, since the interface mesh drifts under the effects of surface tension, electric field and elastic energy, and the preservation of the consistency between the two grids does not modify the topology of the bulk grid, at certain time steps a mesh smoothing may need to be applied, to avoid overlap between bulk elements. Once the quality of the mesh is compromised and cannot be smoothed any longer, a complete, costly remeshing of the bulk region is required. This is in line with existing front-tracking methods for the approximation of void electro-stress migration. But crucially, the good interface mesh properties for our method mean that the only bulk mesh deformations that can occur within our approximation are due to the physical movement of the void within the conductor. In particular, similarly to the method in [31], the vertices on the discrete interface equidistribute asymptotically. This is a major improvement on existing fitted front-tracking methods, see e.g. [13, 25, 36].

\section{Finite element approximation}

We begin with the finite element approximation for quantities defined over the bulk mesh. Let $0=t_{0}<t_{1}<\ldots<t_{M-1}<t_{M}=T$ be a partitioning of $[0, T]$ into possibly variable time steps $\tau_{m}:=t_{m+1}-t_{m}, m=0, \ldots, M-1$. We set $\tau:=\max _{m=0, \ldots, M-1} \tau_{m}$. Let $\mathcal{T}^{m}$ be a partitioning of $\Omega_{+}^{m}$, a polygonal approximation of $\Omega_{+}\left(t_{m}\right)$, into open disjoint triangles. Let $\Gamma^{m}$ be the inner boundary of $\Omega_{+}^{m}$, so that $\partial \Omega_{+}^{m}=\Gamma^{m} \cup \partial \Omega$. We can now define the standard finite element space of piecewise linear functions:

$$
S^{m}:=\left\{\chi \in C\left(\Omega_{+}^{m}\right):\left.\chi\right|_{o} \text { is linear } \forall o \in \mathcal{T}^{m}\right\}
$$

as well as

$$
S_{g}^{m}:=\left\{\chi \in S^{m}:\left.\chi\right|_{\partial_{2}^{ \pm} \Omega}=g^{ \pm}\right\} \quad \text { and } \quad S_{0}^{m}:=\left\{\chi \in S^{m}:\left.\chi\right|_{\partial_{2}^{ \pm} \Omega}=0\right\} .
$$

In addition, we can define the standard finite element space of vector-valued, piecewise quadratic functions:

$$
\underline{Q}^{m}:=\left\{\vec{q} \in C\left(\Omega_{+}^{m}, \mathbb{R}^{2}\right):\left.\vec{q}\right|_{o} \text { is quadratic } \forall o \in \mathcal{T}^{m}\right\},
$$

as well as

$$
\underline{\underline{Q}}^{m}:=\left\{\vec{q} \in \underline{Q}^{m}: \int_{\Omega_{+}^{m}} \vec{q} \cdot \vec{v} \mathrm{~d} x=0 \quad \forall \vec{v} \in \underline{\mathrm{RM}}\right\} .
$$

We introduce now the finite element spaces needed for the approximation of quantities on the moving boundary $\Gamma(t)$. Recall that $\Gamma^{m}$ is by definition the inner boundary of $\Omega_{+}^{m}$ : it is therefore a polygonal 
curve, approximating the closed surface $\Gamma\left(t_{m}\right), m=0, \ldots, M$. Precisely, $\Gamma^{m}=\bigcup_{j=1}^{J} \overline{\sigma_{j}^{m}}$ where $\left\{\sigma_{j}^{m}\right\}_{j=1}^{J}$ is a family of segments, i.e. $\sigma_{j}^{m}=\left[\vec{q}_{j-1}^{m}, \vec{q}_{j}^{m}\right]$ with $\left\{\vec{q}_{j}^{m}\right\}_{j=1}^{J}$ denoting the vertices of $\Gamma^{m}$ and $\vec{q}_{J}^{m}=\vec{q}_{0}^{m}$. Then the necessary finite element spaces can be defined as follows:

$$
\begin{aligned}
W^{m} & :=\left\{\chi \in C\left(\Gamma^{m}\right):\left.\chi\right|_{\sigma_{j}^{m}} \text { is linear } \forall j=1, \ldots, J\right\}, \\
\underline{V}^{m} & :=\left\{\vec{\chi} \in C\left(\Gamma^{m}, \mathbb{R}^{2}\right):\left.\vec{\chi}\right|_{\sigma_{j}^{m}} \text { is linear } \forall j=1, \ldots, J\right\} .
\end{aligned}
$$

Let $\left\{\phi_{j}^{m}\right\}_{j=1}^{J}$ denoting the standard basis functions of $W^{m}$. For scalar and vector functions $f, g \in$ $L^{2}\left(\Gamma^{m}, \mathbb{R}^{(2)}\right)$ we introduce the $L^{2}$ inner product $\langle\cdot, \cdot\rangle_{\Gamma^{m}}$ over the polygonal curve $\Gamma^{m}$ as follows:

$$
\langle f, g\rangle_{\Gamma^{m}}:=\int_{\Gamma^{m}} f \cdot g \mathrm{~d} s .
$$

In addition, if $f, g$ are piecewise continuous, with possible jumps at the nodes $\left\{\vec{q}_{j}^{m}\right\}_{j=1}^{J}$, we define the mass lumped inner product $\langle\cdot, \cdot\rangle_{\Gamma^{m}}^{h}$ as

$$
\langle f, g\rangle_{\Gamma^{m}}^{h}:=\frac{1}{2} \sum_{j=1}^{J}\left|\sigma_{j}^{m}\right|\left[(f \cdot g)\left(\vec{q}_{j-1}^{m,+}\right)+(f \cdot g)\left(\vec{q}_{j}^{m,-}\right)\right],
$$

where we define $f\left(\vec{q}_{j-1}^{m,+}\right):=\lim _{\sigma_{j}^{m} \ni \vec{p} \rightarrow \vec{q}_{j-1}^{m}} f(\vec{p})$ and $f\left(\vec{q}_{j}^{m,-}\right):=\lim _{\sigma_{j}^{m} \ni \vec{p} \rightarrow \vec{q}_{j}^{m}} f(\vec{p})$. In addition, we introduce the outward unit normal $\vec{\nu}^{m}$ to $\Gamma^{m}$; that is,

$$
\vec{\nu}_{j}^{m}=\left.\vec{\nu}^{m}\right|_{\sigma_{j}^{m}}:=-\frac{\left(\vec{q}_{j}^{m}-\vec{q}_{j-1}^{m}\right)^{\perp}}{\left\|\vec{q}_{j}^{m}-\vec{q}_{j-1}^{m}\right\|},
$$

where $\cdot{ }^{\perp}$ acting on $\mathbb{R}^{2}$ denotes clockwise rotation by $\frac{\pi}{2}$.

We note that $\left.\overrightarrow{i d}\right|_{\Gamma^{m}} \in \underline{V}^{m}, m \geq 0$, where $\left.\overrightarrow{i d}\right|_{\Gamma^{m}}$ denotes the identity function on $\Gamma^{m}$. For ease of notation, we will denote $\left.\overrightarrow{i d}\right|_{\Gamma^{m}}$ also by $\vec{X}^{m}$. Then we parameterise the new interface $\Gamma^{m+1}$ with the help of a parameterisation $\vec{X}^{m+1} \in \underline{V}^{m}$.

We propose the following finite element approximation of (1), (3) and (7): Given $\Gamma^{0}$, a polygonal approximation of $\Gamma(0)$, for $m=0, \ldots, M-1$ find functions $\left(\Phi^{m+1}, \vec{U}^{m+1}, \vec{X}^{m+1}, \kappa^{m+1}\right) \in S_{g}^{m} \times \underline{\widehat{Q}}^{m} \times$ $\underline{V}^{m} \times W^{m}$ such that

$$
\begin{array}{cc}
\int_{\Omega_{+}^{m}} \nabla \Phi^{m+1} \cdot \nabla \psi \mathrm{d} x=0 & \forall \psi \in S_{0}^{m}, \\
\int_{\Omega_{+}^{m}} \mathcal{C} \underline{\underline{\mathcal{E}}}\left(\vec{U}^{m+1}\right): \underline{\underline{\mathcal{E}}}(\vec{\zeta}) \mathrm{d} x=\int_{\partial \Omega} \vec{g} \cdot \vec{\zeta} \mathrm{d} s & \forall \vec{\zeta} \in \underline{Q}^{m}, \\
\left\langle\frac{\vec{X}^{m+1}-\vec{X}^{m}}{\tau_{m}}, \chi \vec{\nu}^{m}\right\rangle_{\Gamma^{m}}^{h}-\alpha_{1}\left\langle\kappa_{s}^{m+1}, \chi_{s}\right\rangle_{\Gamma^{m}}=-\left\langle\left(\alpha_{2} \Phi^{m+1}+\alpha_{3} E\left(\vec{U}^{m+1}\right)\right)_{s}, \chi_{s}\right\rangle_{\Gamma^{m}} \forall \chi \in W^{m}, \\
\left\langle\kappa^{m+1} \vec{\nu}^{m}, \vec{\eta}\right\rangle_{\Gamma^{m}}^{h}+\left\langle\vec{X}_{s}^{m+1}, \vec{\eta}_{s}\right\rangle_{\Gamma^{m}}=0 & \forall \vec{\eta} \in \underline{V}^{m} .
\end{array}
$$

Then set $\Gamma^{m+1}=\vec{X}^{m+1}\left(\Gamma^{m}\right)$ and find a suitable triangulation $\mathcal{T}^{m+1}$ of the domain $\Omega_{+}^{m+1}$ with boundary $\partial \Omega_{+}^{m+1}=\Gamma^{m+1} \cup \partial \Omega$. The latter aspect is described in more detail in $\S 4.1$, below. We note that the novel variational approximation of the curvature in (17d) leads to equidistributed mesh points on $\Gamma^{m}$ in practice; see [31] for details. In addition, it is worth noting that the weak formulation of (1), (3) and (7), on which (17) is based, can be derived from (1), (2), (3a) and (7a) respectively, by multiplying with a suitable test function and then performing integration by parts. Then (17a) is a standard finite element approximation of $(3),(17 \mathrm{~b})$ is a standard finite element approximation of $(7)$, while $(17 \mathrm{c}),(17 \mathrm{~d})$ for the case $\alpha_{2}=\alpha_{3}=0$ collapse to the scheme introduced in [4] for (1) with $\alpha_{2}=\alpha_{3}=0$, i.e. for the geometric evolution law of surface diffusion. 
The following theorem establishes the existence and uniqueness of a discrete solution to (17).

THEOREM. 3.1. There exists a unique solution $\left(\Phi^{m+1}, \vec{U}^{m+1}, \vec{X}^{m+1}, \kappa^{m+1}\right) \in S_{g}^{m} \times \underline{\widehat{Q}}^{m} \times \underline{V}^{h} \times W^{h}$ to the system (17). Moreover, if $\alpha_{2}=\alpha_{3}=0$ then it holds that

$$
\left|\Gamma^{m+1}\right|+\alpha_{1} \tau_{m}\left\langle\kappa_{s}^{m+1}, \kappa_{s}^{m+1}\right\rangle_{\Gamma^{m}} \leq\left|\Gamma^{m}\right| .
$$

Proof. We first notice that the equations for $\Phi^{m+1}, \vec{U}^{m+1}$ and $\left(\vec{X}^{m+1}, \kappa^{m+1}\right)$ decouple. The existence of a unique solution for (17a) is trivial. As (17b) is a linear finite dimensional system, existence of $\vec{U}^{m+1}$ follows from uniqueness. It follows from (5) and a Korn's inequality, see e.g. [29, p. 79], that

$$
\int_{\Omega_{+}^{m}} \mathcal{C} \underline{\underline{\mathcal{E}}}(\vec{U}): \underline{\underline{\mathcal{E}}}(\vec{U}) \mathrm{d} x \geq m_{\mathcal{C}} \int_{\Omega_{+}^{m}} \underline{\underline{\mathcal{E}}}(\vec{U}): \underline{\underline{\mathcal{E}}}(\vec{U}) \mathrm{d} x \geq C\|\vec{U}\|_{H^{1}\left(\Omega_{+}^{m}\right)}^{2} \quad \forall \vec{U} \in \underline{\widehat{Q}}^{m} .
$$

Hence we have existence and uniqueness of $\vec{U}^{m+1} \in \widehat{\widehat{Q}}^{m}$ solving (17b). The existence of a solution $\left(\vec{X}^{m+1}, \kappa^{m+1}\right)$ for $(17 \mathrm{c}),(17 \mathrm{~d})$ also follows from uniqueness. To prove the latter we need to show that the only solution to the homogeneous system is the zero solution, and this can be shown as in [31, Theorem 3.1], on noting that the assumption $(\mathcal{A})$ there is trivially satisfied because $\Gamma^{m}$, as the inner boundary of $\Omega_{+}^{m}$, can have no self-intersections.

The stability result (18) can be obtained by choosing $\chi=\kappa^{m+1}$ in (17c) and $\vec{\eta}=\vec{X}^{m+1}-\vec{X}^{m}$ in (17d), on noting that

$$
\left\langle\vec{X}_{s}^{m+1}, \vec{X}_{s}^{m+1}-\vec{X}_{s}^{m}\right\rangle_{\Gamma^{m}}=\left|\Gamma^{m+1}\right|-\left|\Gamma^{m}\right|
$$

see [4, Theorem 2.3] for details.

We note that the stability result (18) for $\alpha_{2}=\alpha_{3}=0$ is the direct discrete analogue of (13). We are not able to prove a discrete analogue of (12) in the case $\alpha_{2}=0$ and $\alpha_{3}>0$. But in practice we never encountered any difficulties, and our discrete solutions always decreased the energy. See e.g. Figure 6 below.

REMARK. 3.1. Clearly, in the case $\alpha_{3}=0$ the systems of equations (17a), (17c), (17d) are very close to the unfitted scheme [31, Eq. (10)]. The only differences are that in (17a) we integrate over the true exterior $\Omega_{+}^{m}$ of $\Gamma^{m}$, rather than over the approximation $\Omega_{+}^{m, h}$, and that on the right hand side of (17c) we use $\Phi^{m+1}$ rather than the Lagrange interpolant $\pi^{h} \Phi^{m+1}$. Of course, both are direct consequences of the fitted approach employed in this paper.

It is straightforward to extend the unfitted scheme [31, Eq. (10)] to the case $\alpha_{3}>0$. The discrete displacement $\vec{U}^{m+1}$ would be computed with the analogue of (17b), but integrated over $\Omega_{+}^{m, h}$. In addition, the right hand side of the evolution equation needs the additional term

$$
-\alpha_{3}\left\langle\left(\pi^{h}\left[E\left(\vec{U}^{m+1}\right)\right]\right)_{s}, \chi_{s}\right\rangle_{\Gamma^{m}}
$$

But the resulting unfitted scheme does not work very well in practice. This is most likely due to the fact that $\Omega_{+}^{m, h}$ is given as a union of bulk mesh elements, and so it has a very rough boundary $\partial \Omega_{+}^{m, h}$. In addition, the vertex-based evaluation of the elastic energy in (19) is far less accurate than the edgebased evaluation employed in (1\%c). In particular, we note that $\pi^{h}\left[E\left(\vec{U}^{m+1}\right)\right]$ is piecewise linear, while $E\left(\vec{U}^{m+1}\right)$ is piecewise quadratic. The fact that we can reliably deal with $\alpha_{3}>0$ only within the fitted approach is one of the main motivations for the presented paper.

REMARK. 3.2. Similarly to [31, §3.2], it is worthwhile to consider a continuous-in-time semidiscrete variant of our fully discrete scheme (17). Completely analogous to the results presented in [31], it is then possible to show that the semidiscrete scheme satisfies the natural discrete analogue to the continuous area preservation property (11). Moreover, it can be shown that the vertices of the discrete interface equidistribute, see [31, Theorem 3.2] for details. While it does not seem possible to prove the equidistribution property for the fully discrete scheme (17), in practice we observe that the vertices on $\Gamma^{m}$ asymptotically equidistribute. 


\section{Solution method}

Due to the special structure of the system (17), the equations for $\Phi^{m+1}, \vec{U}^{m+1}$ and $\left(\vec{X}^{m+1}, \kappa^{m+1}\right)$ decouple. In practice, we can find the unique solution to (17) as follows. First we find $\Phi^{m+1} \in S_{g}^{m}$ such that

$$
\Theta_{m} \Phi^{m+1}=0,
$$

where $\Theta_{m} \in \mathbb{R}^{K \times K}$ is the standard stiffness matrix for the Laplacian on $\Omega_{+}^{m}$, i.e.

$$
\left[\Theta_{m}\right]_{k l}:=\int_{\Omega_{+}^{m}} \nabla \psi_{k}^{m} \cdot \nabla \psi_{l}^{m} \mathrm{~d} x
$$

where $\left\{\psi_{k}^{m}\right\}_{k=1}^{K}$ are the basis functions of the unconstrained finite element space $S^{m}$. In the above we have ignored the effect of the Dirichlet boundary conditions. Hence, in practice, $\Theta_{m}$ and the corresponding right-hand side in (20) need to be adjusted appropriately in order to include the Dirichlet boundary conditions. For the solution of (20) we use the sparse factorisation package UMFPACK (see [15]) in practice.

We proceed with finding $\vec{U}^{m+1}$ such that

$$
\Xi_{m} \vec{U}^{m+1}=G^{m}
$$

where $\Xi_{m} \in \mathbb{R}^{L \times L}$ is the matrix of the elasticity operator on $\Omega_{+}^{m}$, i.e.

$$
\left[\Xi_{m}\right]_{k l}:=\int_{\Omega_{+}^{m}} \mathcal{C} \underline{\underline{\mathcal{E}}}\left(\vec{\xi}_{k}^{m}\right): \underline{\underline{\mathcal{E}}}\left(\vec{\xi}_{l}^{m}\right) \mathrm{d} x
$$

and $G^{m}$ is the right-hand side involving the boundary forcing term, i.e.

$$
\left[G^{m}\right]_{k}:=\int_{\partial \Omega} \vec{g} \cdot \vec{\xi}_{k}^{m} \mathrm{~d} s
$$

Here $\left\{\vec{\xi}_{k}^{m}\right\}_{k=1}^{L}$ are the basis functions of the finite element space $\underline{Q}^{m}$. Therefore, in practice we assemble and solve (21) over $\underline{Q}^{m}$ and not over $\widehat{Q}^{m}$. Recalling (9), such a square system is not invertible, with the kernel of $\Xi_{m}$ corresponding to the finite element functions in RM. Therefore we make use of the sparse factorisation package SuiteSparseQR, which performs a QR factorisation of the noninvertible system (21) and returns a least-square solution; see [16] for details. The solution vector $\vec{U}^{m+1}$ is not guaranteed, in general, to belong to $\widehat{Q}^{m}$. However, recalling that in (17c) we require only the elastic energy density $E\left(\vec{U}^{m+1}\right)$ and not the displacement field $\vec{U}^{m+1}$ itself, there is no need to project $\vec{U}^{m+1}$ onto $\widehat{Q}^{m}$.

Having obtained $\Phi^{m+1}$ from (20) and $\vec{U}^{m+1}$ from (21), we proceed with solving the equations (17c), (17d), which give rise to the following linear system of equations, where we define $\delta \vec{X}^{m+1}=\vec{X}^{m+1}-\vec{X}^{m}$. Find $\delta \vec{X}^{m+1} \in \underline{V}^{m}$ and $\kappa^{m+1} \in W^{m}$ such that

$$
\left(\begin{array}{cc}
\alpha_{1} A_{m} & -\frac{1}{\tau_{m}} \vec{N}_{m}^{T} \\
\vec{N}_{m} & \vec{A}_{m}
\end{array}\right)\left(\begin{array}{c}
\kappa^{m+1} \\
\delta \vec{X}^{m+1}
\end{array}\right)=\left(\begin{array}{c}
f^{m+1} \\
-\vec{A}_{m} \vec{X}^{m}
\end{array}\right) .
$$

In the above, we have introduced the matrices $\vec{N}_{m} \in\left(\mathbb{R}^{2}\right)^{J \times J}, A_{m} \in \mathbb{R}^{J \times J}$ and $\vec{A}_{m} \in\left(\mathbb{R}^{2 \times 2}\right)^{J \times J}$, with entries

$$
\left[\vec{N}_{m}\right]_{k l}:=\left\langle\phi_{k}^{m}, \phi_{l}^{m} \vec{\nu}^{m}\right\rangle_{\Gamma^{m}}^{h}, \quad\left[A_{m}\right]_{k l}:=\left\langle\left(\phi_{k}^{m}\right)_{s},\left(\phi_{l}^{m}\right)_{s}\right\rangle_{\Gamma^{m}},
$$

where we recall that $\left\{\phi_{j}^{m}\right\}_{j=1}^{J}$ are the basis functions of $W^{m}$. In addition, $\left[\vec{A}_{m}\right]_{k l}:=\left[A_{m}\right]_{k l} \overrightarrow{I d}$, where $\overrightarrow{I d} \in \mathbb{R}^{2 \times 2}$ is the identity matrix, and

$$
\left[f^{m+1}\right]_{k}:=\left\langle\left(\alpha_{2} \Phi^{m+1}+\alpha_{3} E\left(\vec{U}^{m+1}\right)\right)_{s},\left(\phi_{k}^{m}\right)_{s}\right\rangle_{\Gamma^{m}} .
$$

The block (22) can be solved either with a sparse factorisation package such as UMFPACK, or with a 
preconditioned BiCGSTAB iterative solver, and we refer to [31] for more details.

\subsection{Mesh operations}

Our fitted finite element approximation (17) is based on triangulations $\mathcal{T}^{m}$ of the discrete conducting regions $\Omega_{+}^{m}$, which vary in time. In addition, recall that $\Gamma^{m}$ is by definition the inner boundary of $\Omega_{+}^{m}$. In our implementation this relationship between the interface mesh $\Gamma^{m}$ and the bulk triangulation $\mathcal{T}^{m}$ is realised with the help of an index map that stores a list of bulk mesh vertices, and their connectivities, that make up the interface $\Gamma^{m}$. This map only needs to be computed once, at time $t_{0}$.

We use an adaptive mesh for $\Omega_{+}^{0}$, where we resolve the regions close to $\Gamma^{0}$ much finer than far away from the interface. In particular, we choose two integer parameters $N_{c}>N_{f}$ to be the number of subdivisions on $\partial^{ \pm} \Omega$ and $\Gamma^{0}$, respectively. This mimics the adaptive meshes used in our unfitted approach in [31]. We then pass these two parameters to the mesh generator GMSH (see [19] for details), which generates a Delaunay triangulation with the desired mesh width on $\partial^{ \pm} \Omega$ and $\Gamma^{0}$.

Once the electro-stress migration problem (17) is solved, we can use the value of $\vec{X}^{m+1}$ at all the vertices of the interface grid in order to update the position of the corresponding vertices of the bulk grid. In theory, the remaining bulk vertices could be left as they are. However, in time the movement of the void will lead to bulk elements being deformed or to overlap. Therefore, the application of some smoothing technique is required, in order to prevent the bulk grid from deteriorating too quickly. To this end, we make use of the so-called linear-elastic smoothing; see [30, 23, 22], where this technique was applied to aerodynamic design optimisation and viscous layer insertion for fluid dynamics problems, respectively. Linear elasticity relationships, borrowed from structural mechanics, provide the governing partial differential equations for smoothly relocating the position of the vertices of the existing mesh. Precisely, we want to find the displacement field $\vec{\Upsilon}$, which is the solution to the following problem:

$$
\begin{array}{lll}
\nabla \cdot(2 \underline{\underline{\mathcal{E}}}(\vec{\Upsilon})+\operatorname{Tr}(\underline{\underline{\mathcal{E}}}(\vec{\Upsilon}))) & =\overrightarrow{0} & \text { in } \Omega_{+}^{m}, \\
\vec{\Upsilon} & =\delta \vec{X}^{m+1} & \text { on } \Gamma^{m}, \\
\vec{\Upsilon} \cdot \vec{\nu}_{\partial \Omega} & =0 & \text { on } \partial \Omega,
\end{array}
$$

i.e. a linear elasticity problem with Lamé moduli $\mu=\lambda=1$. The Dirichlet boundary condition (23b) prescribes a specific displacement on the interface, which we naturally choose to be the variation $\delta \vec{X}^{m+1}$ from (22). The free-slip boundary condition (23c) prevents vertices on the outer boundary from moving away from it. In practice we approximate (23) with the help of piecewise linear finite elements and solve the resulting system of linear equations with the sparse factorisation package UMFPACK. The obtained

discrete variant of $\vec{\Upsilon}$, at every vertex of the current bulk grid, then represents the variation in their position that we compute and apply at every time step to obtain a smoother grid.

Smoothing alone, however, does not prevent the bulk grid from deteriorating in the long run. This is due to the physical motion of the void through the conductor. In order to assess the bulk mesh quality, we make of use of a very simple test: we check all the angles of all the triangles of the smoothed mesh, and if we find any angle $\theta \leq 20^{\circ}$ or $\theta \geq 130^{\circ}$, we then remesh the conducting region $\Omega_{+}^{m}$ altogether. The remeshing is performed with the mesh generator GMSH, which can preserve the position of the vertices on both the inner and outer boundary of $\Omega_{+}^{m}$.

\section{Numerical experiments}

We implement our finite element approximation within the framework of the C++-based software DUNE, see [11, 10], and we employ the Alberta grid manager, see [34]. Unless stated otherwise, we use uniform time steps $\tau_{m}=\tau, m=0, \ldots, M-1$, for all the numerical experiments in this section. As we will compare our numerical results to the phase field computations in [9, 3], we also fix $\alpha_{1}=\frac{1}{16} \pi^{2}$. When stress-migration is considered, we assume that (6) holds and put $\alpha_{3}=\frac{1}{8} \pi$ throughout this section. On recalling Remark 3.2, we observe that the fully discrete scheme (17) approximately preserves the area of $\Omega_{+}^{m}$. In fact, in all of the numerical simulations presented in this paper, the observed relative area loss was always less than $0.001 \%$.

We begin with a convergence experiment for the true solution (14), and compare the results with the corresponding experiments in [31, Table 1]. Recall that (14) describes a circular void that moves 
at constant speed through an infinite conductor. Here only electro-migration is considered, and so $\alpha_{3}=0$. We chose the following parameters: $L_{1}=1.5, L_{2}=0.5, \alpha_{2}=3 \pi^{2}$. The initial geometry is a circle with radius $R=0.25$ and centre $z=(-0.5,0)$, while $T=2 \times 10^{-3}$. Following [31], we define the error $\mathcal{E}_{\Gamma}=\|\vec{X}-\vec{x}\|_{L^{\infty}}:=\max _{m=1, \ldots, M}\left\|\overrightarrow{X^{m}}-\vec{x}\left(\cdot, t_{m}\right)\right\|_{L^{\infty}}$, where $\left\|\overrightarrow{X^{m}}-\vec{x}\left(\cdot, t_{m}\right)\right\|_{L^{\infty}}:=$ $\left.\max _{i=1, \ldots, N}\left\{\min _{\vec{y} \in \Gamma(t)} \mid \vec{X}^{m}\left(\vec{q}_{i}^{m}\right)-\vec{x}\left(\vec{y}, t_{m}\right)\right) \mid\right\}$ between $\vec{X}$ and the true solution on the interval [0,T]. In addition, we define the error $\mathcal{E}_{\text {bulk }}:=\max _{i=1, \ldots, M}\left\|\Phi^{m}-\phi\left(\cdot, t_{m}\right)\right\|_{H^{1}\left(\Omega_{+}^{m-1}\right)}$ between $\Phi$ and the exact electric potential $\phi$ on the interval $[0, T]$. Since the exact solution is known, it is worth calculating an experimental order of convergence. To this end, we define

$$
h_{\Gamma}^{(i)}=\frac{2 L_{2}}{J_{i}} \quad \text { with } J_{i}:=N_{f}=16 N_{c}=2^{7+i}, \quad i=0, \ldots, 3 .
$$

The experimental orders of convergence EOC are computed as $\log \left(\frac{\mathcal{E}^{(i-1)}}{\mathcal{E}^{(i)}}\right) / \log 2$. The corresponding errors are listed in Table 1, where it appears that we observe a convergence of $\mathcal{O}\left(h^{2}\right)$ in the measured error for the interface, and $\mathcal{O}(h)$ for the $H^{1}$-error for the approximation of the electric potential in the bulk. We also note that in all the four cases $i=0, \ldots, 3$ it was necessary to remesh the conducting region only once. The computational overhead for this remeshing routine is minimal, compared to the total CPU time. For example, for the case $i=3$ the remeshing step took ten seconds. For ease of comparison, we

\begin{tabular}{|c|c|c|c|c|c|c|c|rr|}
\hline$i$ & $\tau \cdot 10^{6}$ & $h_{\Gamma} \cdot 10^{3}$ & $h_{\text {bnd }} \cdot 10^{2}$ & $\mathcal{E}_{\text {bulk }} \cdot 10^{2}$ & $\mathrm{EOC}_{\text {bulk }}$ & $\mathcal{E}_{\Gamma} \cdot 10^{3}$ & $\mathrm{EOC}_{\Gamma}$ & \multicolumn{2}{|c|}{ CPU time } \\
& & & & & & & & & \\
Total & Smoothing \\
\hline 0 & 8 & 7.81 & 12.5 & 4.369 & - & 2.378 & - & $15 \mathrm{~s}$ & $2 \mathrm{~s}$ \\
1 & 2 & 3.91 & 6.25 & 2.201 & 0.99 & 0.625 & 1.93 & $201 \mathrm{~s}$ & $39 \mathrm{~s}$ \\
2 & 0.5 & 1.95 & 3.13 & 1.123 & 0.97 & 0.155 & 2.01 & $3287 \mathrm{~s}$ & $764 \mathrm{~s}$ \\
3 & 0.125 & 0.977 & 1.56 & 0.564 & 0.99 & 0.0399 & 1.96 & $72551 \mathrm{~s}$ & $22143 \mathrm{~s}$ \\
\hline
\end{tabular}

Table 1: Results of the convergence test for the fitted case, with $h_{\Gamma}$ and $h_{\mathrm{bnd}}$ being the width of the mesh on the inner and the outer bondary of $\Omega$, respectively. Note that the average number of bulk degrees of freedom for the four runs were 886 , 3125,12033 and 48977, respectively.

recall the results obtained in our previous work [31] in Table 2, where here we add more information about the total CPU time required to complete the simulations. With the unfitted approach, a convergence of

\begin{tabular}{|c|c|c|c|c|c|c|c|r|}
\hline$i$ & $\tau \cdot 10^{6}$ & $h_{\Gamma} \cdot 10^{3}$ & $h_{\text {bnd }} \cdot 10^{2}$ & $\mathcal{E}_{\text {bulk }} \cdot 10^{2}$ & $\mathrm{EOC}_{\text {bulk }}$ & $\mathcal{E}_{\Gamma} \cdot 10^{3}$ & $\mathrm{EOC}_{\Gamma}$ & CPU time \\
\hline 0 & 8 & 7.81 & 12.5 & 9.037 & - & 16.459 & - & $18 \mathrm{~s}$ \\
1 & 2 & 3.91 & 6.25 & 5.919 & 0.61 & 7.556 & 1.12 & $174 \mathrm{~s}$ \\
2 & 0.5 & 1.95 & 3.13 & 3.650 & 0.69 & 3.474 & 1.12 & $2098 \mathrm{~s}$ \\
3 & 0.125 & 0.977 & 1.56 & 2.143 & 0.77 & 1.524 & 1.19 & $37861 \mathrm{~s}$ \\
\hline
\end{tabular}

Table 2: Results of the convergence test for the unfitted case, reproduced from [31, Table 1]. Note that the average number of bulk degrees of freedom for the four runs were 990, 2296, 5987 and 17677, respectively.

at least $\mathcal{O}(h)$ in $\mathcal{E}_{\Gamma}$ and at least $\mathcal{O}\left(h^{\frac{1}{2}}\right)$ in $\mathcal{E}_{\text {bulk }}$ were observed. Hence the fitted method introduced in this paper appears to converge with a faster rate. In addition, we note that in each case the absolute errors in Table 1 are smaller than the corresponding errors in Table 2. However, apart from the very coarse case $i=0$, we note that the fitted method requires more CPU time. This has two reasons. Clearly, the mesh smoothing performed after each time step, which involves solving a discrete variant of (23), contributes to the total CPU time. Moreover, the Delaunay meshes generated with the help of the package GMSH show a smoother transition in density of elements from regions with finer width $h_{\Gamma}$ to regions with coarser width $h_{\mathrm{bnd}}$, compared to the unfitted meshes used in [31]. This results in a larger number of elements far from the interface, compared to the case of meshes with right-angled, isosceles triangles, as used in [31]. This leads to a larger number of bulk degrees of freedom, which in turn increases the CPU time needed to solve the relevant systems of linear equations.

Our next experiment is presented in Figure 2, and involves both electro-migration and stress-migration. 
We chose the following parameters: $L_{1}=2.5, L_{2}=0.5, \alpha_{2}=10 \pi^{2}, \mu=\lambda=0.5, \underline{\underline{S}}=\left(\begin{array}{ll}1 & 0 \\ 0 & 1\end{array}\right), \tau=$ $5 \times 10^{-7}, J=1024, N_{f}=1024, N_{c}=16$. The initial geometry is a circle with radius $R=0.25$ and centre $z=(-1.5,0)$, while $T=3.75 \times 10^{-3}$. The total CPU time was $52526 \mathrm{~s}$, with mesh smoothing and remeshings accounting for $2 \%$ of that time. The remeshing routine was applied ten times. The total number of bulk degrees of freedom (for both $\Phi^{m+1}$ and $\vec{U}^{m+1}$ ) was between 84139 (minimum value) and 91879 (maximum value). Naturally, the number of degrees of freedom for the interface problem (17c), (17d) is given by $3 J$ here and throughout this section. In Figure 2 we plot the results of the simulation at times $t=0,1.25 \times 10^{-3}, 2.5 \times 10^{-3}$ and $T=3.75 \times 10^{-3}$. It can be seen that the void moves through the conductor due to the presence of the electric field. The elastic stress leads to a small tip developing at the front of the void.

In the next experiment we chose $\underline{\underline{S}}=\left(\begin{array}{ll}1 & 0 \\ 0 & 0\end{array}\right), \mu=\lambda=1$, with all the other parameters as in the previous case. The total CPU time was $5244 \overline{3}$ s, with mesh smoothing and remeshings accounting for $2 \%$ of that time. We applied the remeshing routine nine times. The total number of bulk degrees of freedom (for both $\Phi^{m+1}$ and $\vec{U}^{m+1}$ ) was between 83770 (minimum value) and 90511 (maximum value). Results of the simulation are plotted in Figure 3, where we observe that the profile of the moving void is now very different.

Our fourth experiment corresponds to [3, Fig. 1], where a circular void is subject to stress-migration only (i.e., $\left.\alpha_{2}=0\right)$. We chose the following parameters: $L_{1}=L_{2}=0.5, \mu=\lambda=\frac{4}{5 \pi}, \underline{\underline{S}}=\left(\begin{array}{ll}0 & 0 \\ 0 & 1\end{array}\right)$, $\tau=10^{-6}, J=2048, N_{f}=2048, N_{c}=32$. The initial geometry is a circle with radius $R=0.25$ and centre $z=(0,0)$, while $T=0.02$. The total CPU time was $877828 \mathrm{~s}$, with the mesh smoothing accounting for $1.5 \%$ of that time. In this experiment no remeshing was necessary, since the bulk mesh hardly changes after the $1000^{t h}$ time step. The number of degrees of freedom for $\vec{U}^{m+1}$ was 255336 . In Figure 4 we plot the result of the simulation at time $t=T$ and note the good agreement with the results presented in $[3$, Fig. 1].

Our last experiment is presented in Figure 5, where only stress-migration is considered. We chose the following parameters: $L_{1}=L_{2}=0.5, \mu=0.5, \lambda=0, \underline{\underline{S}}=\left(\begin{array}{ll}1 & 0 \\ 0 & 0\end{array}\right), \tau=10^{-6}, J=2048, N_{f}=1024, N_{c}=$ 16. The initial geometry is composed of two circles with radius $R=0.15$ and centres $z_{ \pm}=( \pm 0.22,0)$, while $T=1.5 \times 10^{-3}$. The total CPU time was $78830 \mathrm{~s}$, with mesh smoothing and remeshings accounting for $1.5 \%$ of that time. Here the remeshing routine was applied only once. The number of degrees of freedom for $\vec{U}^{m+1}$ was between 226846 (minimum value) and 244942 (maximum value). In Figure 5 we plot the results of the simulation at times $t=0,5 \times 10^{-4}, 10^{-3}$ and $T=1.5 \times 10^{-3}$. For this experiment we observe that for larger times $T$ a singularity develops. In particular, the interface shows corners and the elastic energy becomes unbounded. This can be seen in Figure 6, where the developing singularity leads to a breakdown of symmetry in the numerical approximation. However, the presented plot of the total discrete energy indicates that the singularity is not due to numerical errors. In fact, the discrete energy is monotonically decreasing, recall also (12). Hence the observed singularity appears to be consistent with the sharp interface model (1), (7) itself.

\section{Conclusion}

We have presented a fitted front-tracking method for the approximation of electro-stress migration using parametric finite elements. The main properties of our method are that we can prove an equidistribution property for the vertices on the discrete interface, and that in the absence of external forces the scheme can be shown to be unconditionally stable. We note that in practice, in all our computations, the method is also stable in the presence of forces due to the applied electric field and to the elastic stresses.

Our presented method in many ways is similar to the scheme in [31], where we introduced an unfitted approach. Let us summarise the main advantages and disadvantages of the two methods. In the unfitted scheme, the bulk mesh is totally independent from the interface approximation. Hence standard refinement and coarsening strategies can be employed for the bulk mesh, and no mesh smoothings or remeshings are necessary. The communication between the bulk mesh and the independent, lower dimensional interface mesh needs to be implemented manually, as this is not part of standard finite element packages. However, once implemented such communication can be done efficiently, and without significant computational overhead, see e.g. [33, 7] for applications in the context of dendritic growth. A disadvantage of the unfitted approach is that without significant effort in the implementation of an 

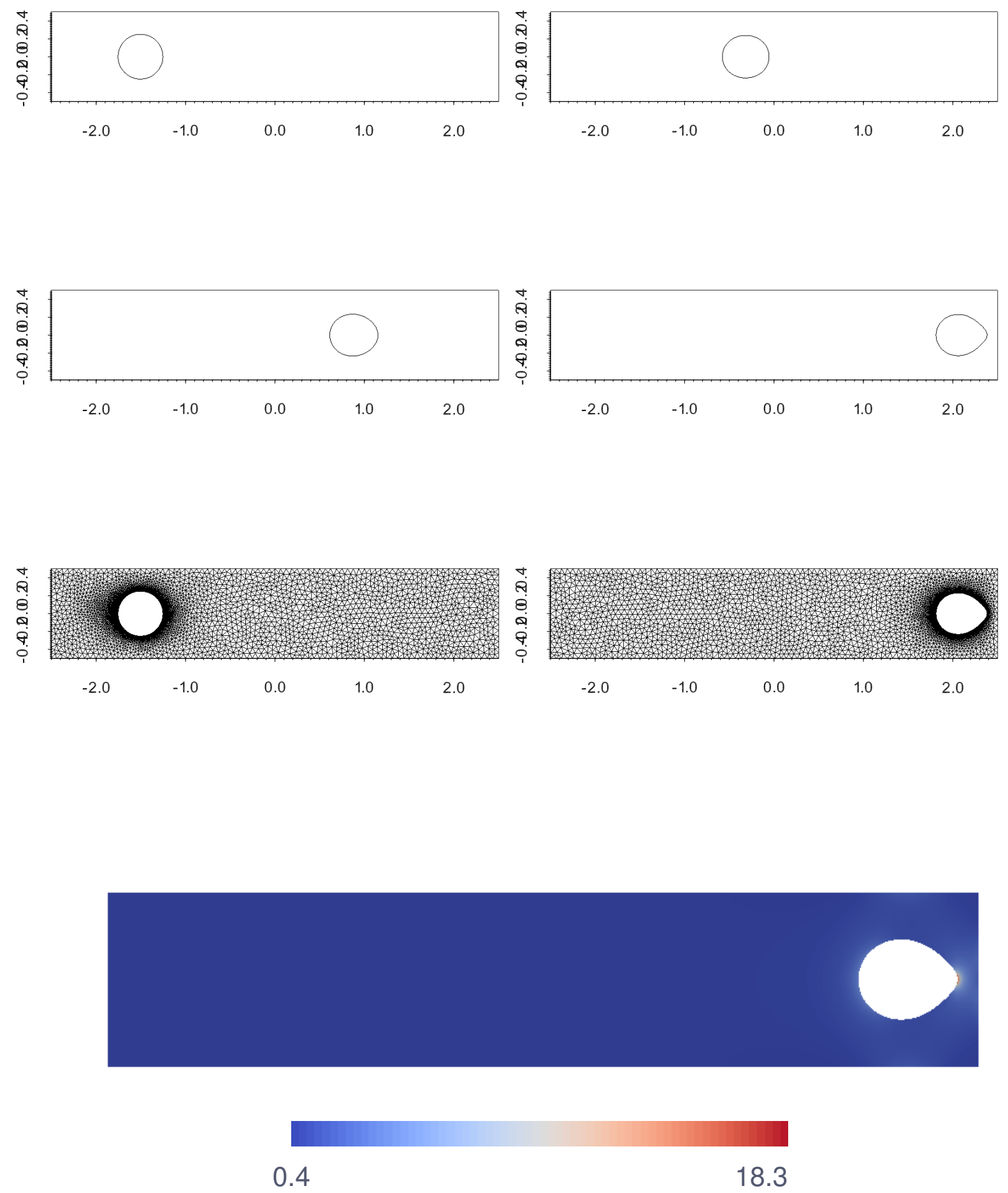

Figure 2: $\left(\alpha_{2}=10 \pi^{2}, \mu=\lambda=0.5, \underline{\underline{S}}=\left(\begin{array}{ll}1 & 0 \\ 0 & 1\end{array}\right)\right)$ Plots of the interface curve at times $t=0,1.25 \times 10^{-3}, 2.5 \times 10^{-3}$ and $T=$ $3.75 \times 10^{-3}$, bulk mesh at time $t=\overline{0}, T$ and the elastic energy at time $t=T$. 

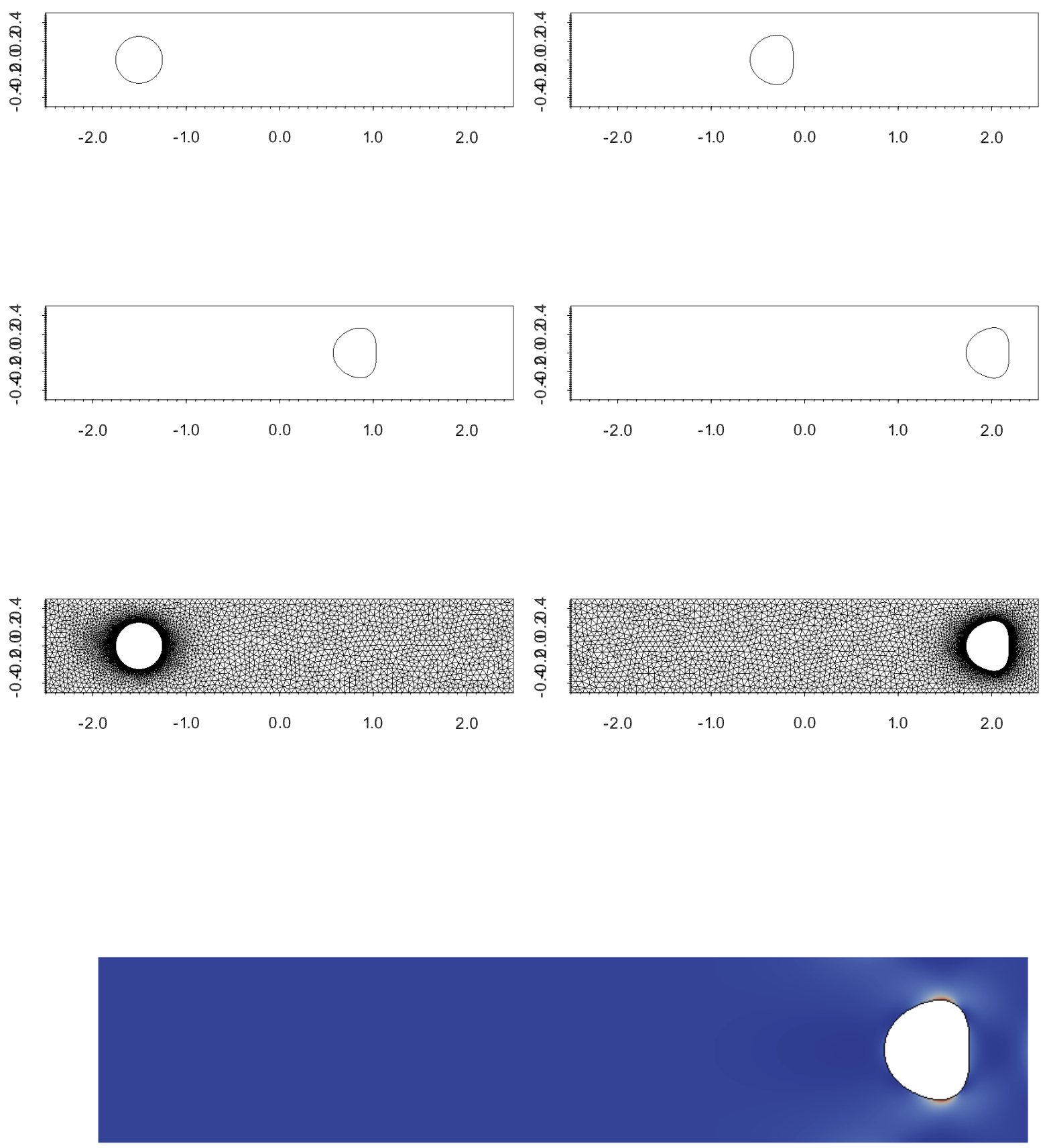

Figure 3: $\left(\alpha_{2}=10 \pi^{2}, \mu=\lambda=1, \underline{\underline{S}}=\left(\begin{array}{ll}1 & 0 \\ 0 & 0\end{array}\right)\right)$ Plots of the interface curve at times $t=0,1.25 \times 10^{-3}, 2.5 \times 10^{-3}$ and $T=$ $3.75 \times 10^{-3}$, bulk mesh at time $t=0, T$ and the elastic energy density at time $t=T$. 

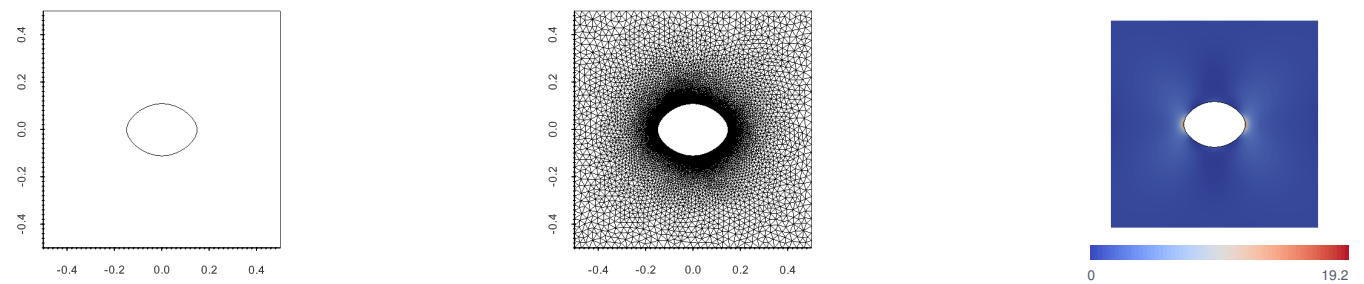

Figure 4: $\left(\mu=\lambda=\frac{4}{5 \pi}, \underline{\underline{S}}=\left(\begin{array}{ll}0 & 0 \\ 0 & 1\end{array}\right)\right)$ Plots of the interface curve, the bulk mesh and the elastic energy density at time $T=0.02$.
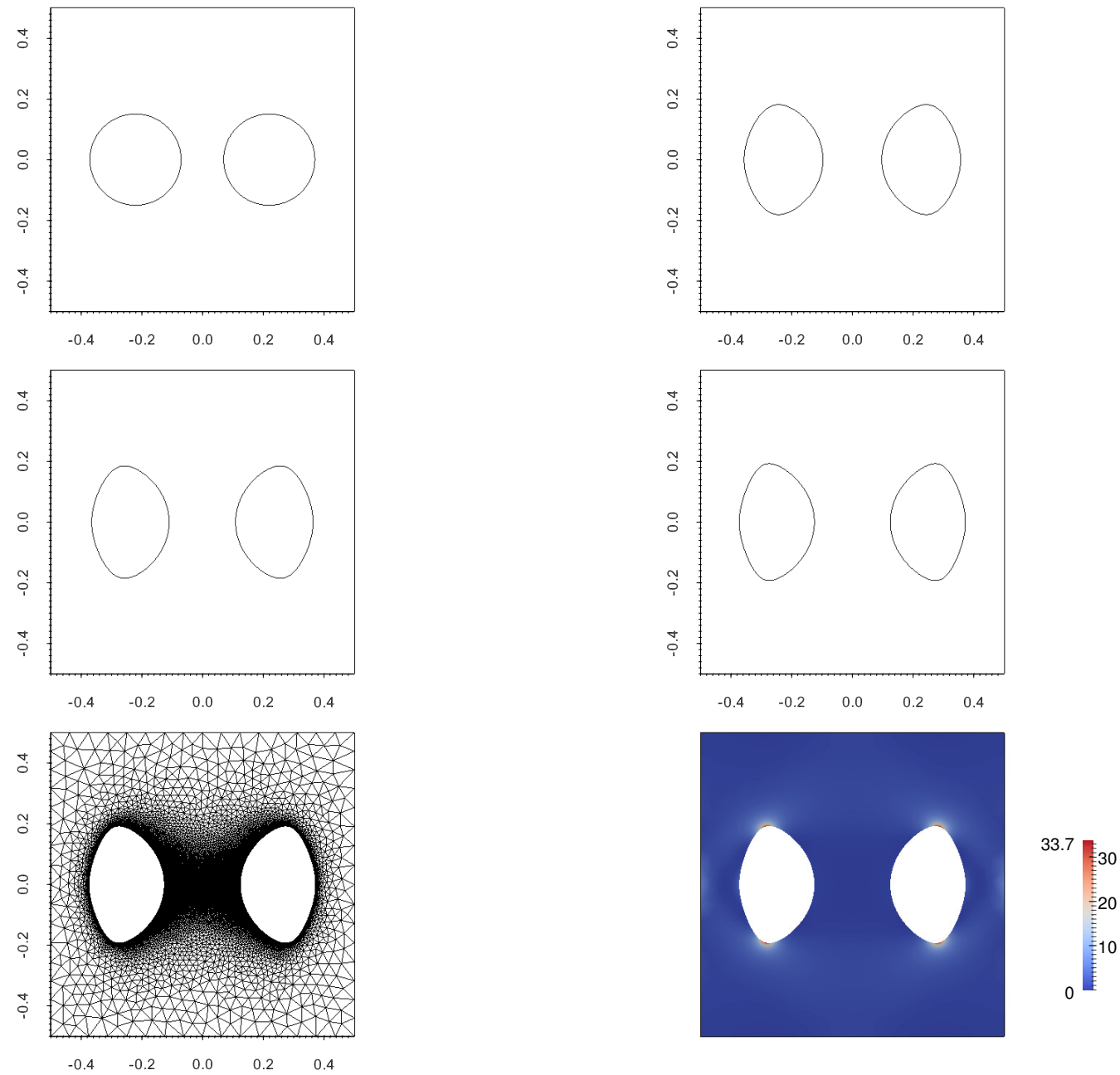

Figure 5: $\left(\mu=0.5, \lambda=0, \underline{S}=\left(\begin{array}{ll}1 & 0 \\ 0 & 0\end{array}\right)\right)$ Plots of the interface curve at times $t=0,5 \times 10^{-4}, 10^{-3}$, and $T=1.5 \times 10^{-3}$, bulk mesh and the elastic energy density at time $t=T$. 


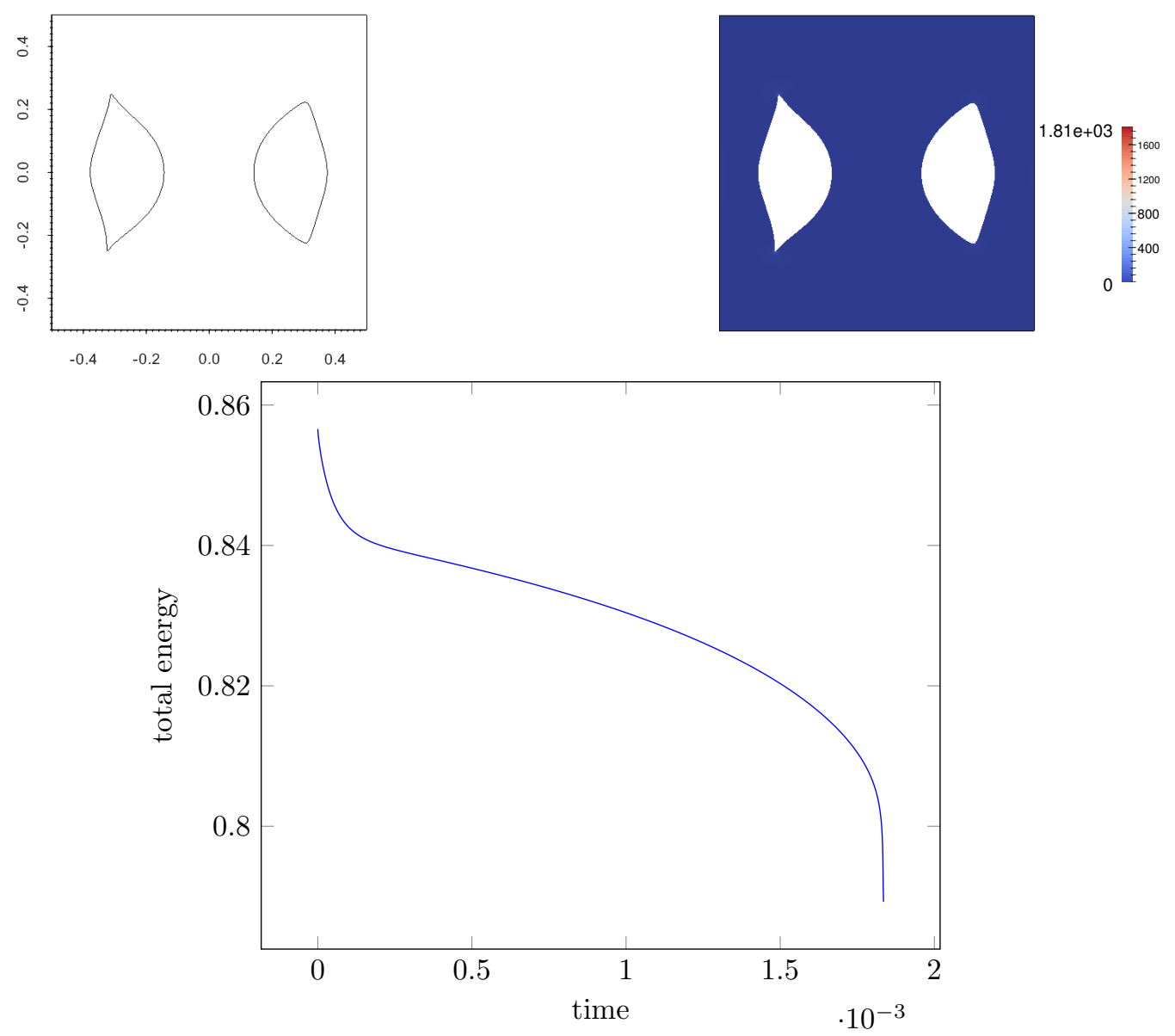

Figure 6: $\left(\mu=0.5, \lambda=0, \underline{\underline{S}}=\left(\begin{array}{ll}1 & 0 \\ 0 & 0\end{array}\right)\right)$ Plots of the interface curve and the elastic energy density at time $t=1.834 \times 10^{-3}$. Below a plot of the total discrete energy $\alpha_{1}\left|\Gamma^{m}\right|+\alpha_{3}\left(\int_{\Omega_{+}^{m}} E\left(\vec{U}^{m+1}\right) \mathrm{d} x-\int_{\partial \Omega} \vec{g} \cdot \vec{U}^{m+1} \mathrm{~d} s\right)$ over time.

approximate exterior, the weak approximation of the natural boundary conditions in (3a) and (7b) introduces additional numerical errors. A computationally intensive strategy, that was not employed in [31], is discussed in [8, Remark 4.2].

The main advantage of the fitted method proposed in this paper, on the other hand, is that the true exterior of the discrete interface is immediately available. Moreover, modern finite element packages provide the implementation of trace finite element spaces on lower dimensional submeshes as standard, see e.g. [24]. In this paper we implemented the necessary trace finite element spaces ourselves, within the framework of DUNE, see [11, 10]. Having the exact exterior of the discrete interface and the exact traces of bulk finite elements on the discrete interface available means that the fitted method is more accurate in practice. The main disadvantage of the fitted method is that the movement of the interface means that the bulk mesh cannot remain static. Rather, we apply mesh smoothing after every time step in order to avoid the creation of very degenerate or even overlapping bulk elements. In our simulations, the mesh smoothing can account for up to $30 \%$ of the total CPU time in the case of electro-migration, while the higher computational demands for the stress-migration problem mean that the mesh smoothing accounts for only up to $2 \%$ of the total CPU times in our electro-stress migration simulations. On top of that, when mesh smoothing alone is not sufficient to improve the quality of the mesh, we need to remesh the conducting region completely. However, compared to the total CPU time spent on solving of the linear systems that arise at each time step, the CPU time spent on remeshings is small.

[1] A. Averbuch, M. Israeli, I. Ravve, I. Yavneh, Computation for electromigration in interconnects of microelectronic devices, J. Comput. Phys. 167 (2) (2001) 316-371. 
[2] E. Bänsch, P. Morin, R. H. Nochetto, A finite element method for surface diffusion: the parametric case, J. Comput. Phys. 203 (1) (2005) 321-343.

[3] J. Barrett, H. Garcke, R. Nürnberg, Finite element approximation of a phase field model for surface diffusion of voids in a stressed solid, Math. Comp. 75 (253) (2006) 7-41.

[4] J. W. Barrett, H. Garcke, R. Nürnberg, A parametric finite element method for fourth order geometric evolution equations, J. Comput. Phys. 222 (1) (2007) 441-467.

[5] J. W. Barrett, H. Garcke, R. Nürnberg, A phase field model for the electromigration of intergranular voids, Interfaces Free Bound. 9 (2007) 171-210.

[6] J. W. Barrett, H. Garcke, R. Nürnberg, On the parametric finite element approximation of evolving hypersurfaces in $\mathbb{R}^{3}$, J. Comput. Phys. 227 (9) (2008) 4281-4307.

[7] J. W. Barrett, H. Garcke, R. Nürnberg, On stable parametric finite element methods for the Stefan problem and the Mullins-Sekerka problem with applications to dendritic growth, J. Comput. Phys. 229 (18) (2010) 6270-6299.

URL http://dx.doi.org/10.1016/j.jcp.2010.04.039

[8] J. W. Barrett, H. Garcke, R. Nürnberg, Finite element approximation of one-sided Stefan problems with anisotropic, approximately crystalline, Gibbs-Thomson law, Adv. Differential Equations 18 (34) (2013) 383-432.

URL http://projecteuclid.org/euclid.ade/1360073021

[9] J. W. Barrett, R. Nürnberg, V. Styles, Finite element approximation of a phase field model for void electromigration, SIAM J. Numer. Anal. 42 (2) (2004) 738-772.

[10] P. Bastian, M. Blatt, A. Dedner, C. Engwer, R. Klöfkorn, R. Kornhuber, M. Ohlberger, O. Sander, A generic grid interface for parallel and adaptive scientific computing. Part II: Implementation and tests in DUNE, Computing 82 (2-3) (2008) 121-138.

[11] P. Bastian, M. Blatt, A. Dedner, C. Engwer, R. Klöfkorn, M. Ohlberger, O. Sander, A generic grid interface for parallel and adaptive scientific computing. Part I: Abstract framework, Computing $82(2-3)(2008)$ 103-119.

[12] D. N. Bhate, A. Kumar, A. F. Bower, Diffuse interface model for electromigration and stress voiding, J. Appl. Phys. 87 (4) (2000) 1712-1721.

[13] A. Bower, L. Freund, Finite element analysis of electromigration and stress induced diffusion in deformable solids, MRS Proceedings 391 (1995) 177-188.

[14] L. J. Cummings, G. Richardson, M. Ben Amar, Models of void electromigration, European J. Appl. Math. 12 (2) (2001) 97-134.

[15] T. A. Davis, Algorithm 832: UMFPACK V4.3-an unsymmetric-pattern multifrontal method, ACM Trans. Math. Softw. 30 (2) (2004) 196-199.

[16] T. A. Davis, Algorithm 915, SuiteSparseQR: Multifrontal multithreaded rank-revealing sparse QR factorization, ACM Transactions on Mathematical Software (TOMS) 38 (1) (2011) 8.

[17] K. Deckelnick, G. Dziuk, C. M. Elliott, Computation of geometric partial differential equations and mean curvature flow, Acta Numer. 14 (2005) 139-232.

URL http://dx.doi.org/10.1017/S0962492904000224

[18] C. M. Elliott, H. Garcke, Existence results for diffusive surface motion laws, Adv. Math. Sci. Appl. 7 (1) (1997) 467-490.

[19] C. Geuzaine, J.-F. Remacle, Gmsh: A 3-D finite element mesh generator with built-in pre-and post-processing facilities, Int. J. Numer. Meth. Eng. 79 (11) (2009) 1309-1331. 
[20] M. E. Gurtin, The linear theory of elasticity, in: S. Flügge, C. Truesdell (eds.), Handbuch der Physik, Vol. VIa/2, Springer-Verlag, Berlin, 1972.

[21] P. S. Ho, Motion of inclusion induced by a direct current and a temperature gradient, J. Appl. Phys. 41 (1970) 64-68.

[22] S. L. Karman, Unstructured viscous layer insertion using linear-elastic smoothing, AIAA J. 45 (1) (2007) 168-180.

[23] S. L. Karman, W. K. Anderson, M. Sahasrabudhe, Mesh generation using unstructured computational meshes and elliptic partial differential equation smoothing, AIAA J. 44 (6) (2006) 1277-1286.

[24] D. Köster, O. Kriessl, K. G. Siebert, Design of finite element tools for coupled surface and volume meshes, Numer. Math. Theory Methods Appl. 1 (3) (2008) 245-274.

[25] O. Kraft, E. Arzt, Electromigration mechanisms in conductor lines: Void shape changes and slit-like failure, Acta Mater. 45 (4) (1997) 1599-1611.

[26] Z. Li, H. Zhao, H. Gao, A Numerical Study of Electro-migration Voiding by Evolving Level Set Functions on a Fixed Cartesian Grid, J. Comput. Phys. 152 (1999) 281-304.

[27] M. Mahadevan, R. M. Bradley, Phase field model of surface electromigration in single crystal metal thin films, Physica D: Nonlinear Phenomena 126 (3) (1999) 201-213.

[28] M. Mahadevan, R. M. Bradley, Simulations and theory of electromigration-induced slit formation in unpassivated single-crystal metal lines, Physical Review B 59 (16) (1999) 11037-11046.

[29] J. Nečas, I. Hlaváček, Mathematical theory of elastic and elasto-plastic bodies: An introduction, Elsevier, Amsterdam, 1981.

[30] E. J. Nielsen, W. K. Anderson, Recent improvements in aerodynamic design optimization on unstructured meshes, AIAA J. 40 (6) (2002) 1155-1163.

[31] R. Nürnberg, A. Sacconi, An unfitted finite element method for the numerical approximation of void electromigration, J. Comp. Appl. Math. 270 (0) (2014) 531-544, Fourth International Conference on Finite Element Methods in Engineering and Sciences (FEMTEC 2013).

[32] S. Osher, J. A. Sethian, Fronts propagating with curvature-dependent speed: Algorithms based on Hamilton-Jacobi formulations, J. Comput. Phys. 79 (1) (1988) 12-49.

[33] A. Schmidt, Computation of three dimensional dendrites with finite elements, J. Comput. Phys. 195 (2) (1996) 293-312.

[34] A. Schmidt, K. G. Siebert, Design of Adaptive Finite Element Software: The Finite Element Toolbox ALBERTA, vol. 42 of Lecture Notes in Computational Science and Engineering, Springer-Verlag, Berlin, 2005.

[35] J. A. Sethian, Level set methods and fast marching methods, vol. 3 of Cambridge Monographs on Applied and Computational Mathematics, 2nd ed., Cambridge University Press, Cambridge, 1999, evolving interfaces in Computational Geometry, Fluid Mechanics, Computer Vision, and Materials Science.

[36] L. Xia, A. Bower, Z. Suo, C. Shih, A finite element analysis of the motion and evolution of voids due to strain and electromigration induced surface diffusion, J. Mech. Phys. Solids 45 (9) (1997) $1473-1493$. 